\title{
THE AMINO-TERMINAL PROPEPTIDE OF TYPE I PROCOLLAGEN IN GROWTH HORMONE-DEFICIENT PATIENTS DURING TRANSITION PERIOD
}

\author{
Mariana Costache-Outas ${ }^{1,2}$, Andra Caragheorgheopol ${ }^{3}$, Camelia Procopiuc $^{3}$, \\ Cristina Dumitrescu ${ }^{3}$, Cosmina Raluca Costache ${ }^{4}$, Simona Fica ${ }^{1,5}$ \\ 1 "Carol Davila" University of Medicine and Pharmacy, Bucharest \\ 2 "Coltea" Clinical Hospital, Bucharest \\ 3 “C.I. Parhon" National Institute of Endocrinology, Bucharest \\ 4 "Dr. Ioan Cantacuzino" Clinical Hospital, Bucharest \\ 5 “Elias” Emergency Hospital, Endocrinoology Department, Bucharest
}

\begin{abstract}
Introduction. Bone mineral accretion continues beyond the attainment of final height during the transition period. Growth hormone deficiency (GHD) appears to have a significant effect on collagen turnover during childhood and less during adulthood. Amino-terminal propeptide of type I collagen (P1NP) is a marker of bone formation with low intra-individual when comparing to IGF1.

Material and method. We evaluated 17 male patients diagnosed with GHD during childhood, after retesting $\mathrm{GH}$ axis in their transition period after at least 3 months from $\mathrm{GH}$ withdrawal. We correlate concentrations of P1NP and IGF1. We determined the predictive value for P1NP in identifying persistent GHD.

Results. We found a strong positive correlation between IGF-1 and P1NP in the group of patients who maintained $\mathrm{GH}$ deficiency as young adults $(r=0.72, \mathrm{Cl}[0.02$ to 0.94$], p=0.046)$. A threshold value for the P1NP of - 0.66 SDS predicts persistence of GHD with a sensitivity of $62.5 \% \mathrm{Cl}$ [24.5 to 91.5$]$, specificity $75 \% \mathrm{Cl}$ [ 47.6 to 92.7] and $A \cup C=0.719 \mathrm{Cl}[0.50881]$. We did not find a significant difference when we compared the AUC for the two parameters $(p=0.29)$.

Conclusion. During the transition period, when the growth velocity is not available anymore, the dynamics of P1NP may be useful in quantifying the effectiveness of GH replacement therapy.
\end{abstract}

Keywords: Collagen markers, P1NP, growth hormone, IGF1, transition period, persistent GHD

\section{Acknowledgements}

This work received partial financial support through the project entitled "CERO-Career profile: Romanian Researcher", grant number POSDRU/159/1.5/S/135760, co-financed by the European Social Fund for Sectoral Operational Programme Human Resources Development 2007-2013.

\section{INTRODUCTION}

Bone mineral accretion continues beyond the attainment of final height during the transition that is defined as the period elapsed since the achievement of final height and the somatic development to complete. Growth hormone (GH) - insulin-like growth factor I (IGF 1) axis has a basic effect on peak bone density which is reached in the midtwenties (1). The ultradian rhythm of GH release has made it difficult to identify precisely and accurately the deficiency state (2). The serum IGF1 and collagen markers increase in response to $\mathrm{GH}(3,4)$. Although IGF1 has been extensively used in clinical settings as a screening test for GH deficiency (GHD), his coefficient of reliability is lower than that of the collagen markers (5). Collagen markers are not considered a diagnostic tool for GHD (6). Serum surrogate markers of collagen synthesis allow the assessment of bone formation $(7,8)$.

The amino-terminal propeptide of type I procollagen is derived from type I procollagen in a stoichiometric fashion and is considered a measure of

Corresponding author:

Mariana Costache-Outas, "Coltea" Clinical Hospital, 1-3 I.C. Bratianu Street, Bucharest

E-mail: costache.mariana@gmail.com 
newly formed type I collagen, the major collagen of the bone.

During childhood growth, surrogate markers of bone formation circulate at higher concentrations than in adulthood and correlate with height velocity $(9,10)$. Their levels vary during puberty and are higher during periods of maximal growth velocity. $\mathrm{GH}$, as well as sexual hormones, influence the levels of bone formation markers (11). GHD appears to have a relevant effect on collagen turnover during childhood and less during adulthood, when the sexual hormones and local factors partially counteract the negative consequences on collagen synthesis of the GH-IGF1 deficiency (12).

There is no data available on surrogate markers of bone turnover and their level depending on $\mathrm{GH}$ status during the transition.

To gain more insight into the value of P1NP measurements in the screening for persistent GHD (pGHD) in the transition, we assessed its concentrations in GHD adolescents and healthy volunteers and we compared them with IGF1 levels. We hypothesized that P1NP is lower in pGHD patients during the transition.

\section{METHODS}

\section{Subjects}

P1NP was evaluated in a cross-sectional study. 17 consecutive male patients with GHD during childhood isolated (CO-IGHD) or as part of multiple pituitary hormone deficiency (MPHD) (4pts), followed within the Pediatric Department of National Institute of Endocrinology, were selected for this study. Part of the present group was used in a previous study to evaluate the persistence of GHD in their transition period.

All patients had the indication of therapy withdrawal for the attainment of final height during the period between September 2008 and December 2013. The CO-IGHD patients were retested for the persistence of GHD in their transition period using insulin-induced hypoglycemia test (ITT) with the cut-off for GH maximal response $6.1 \mathrm{ng} / \mathrm{dL}$. Following ITT, 4 of the 13 patients with CO-IGHD were considered persistent GHD as young adults and together with MPHD patients were included in the pGHD group (unpublished data). A control group of 8 healthy subjects matched for age and the pubertal stage was recruited from the children of the medical personnel of our institute. The sufficient GH group (sGH) included reversible GHD subjects and the controls.
The study was approved by the Department of Pediatrics institutional review board, and written informed consent was obtained from all parents and patients.

\section{Biochemical assays}

Serum IGF1 and P1NP were collected between 8 and 9 am, after overnight fasting. Within the next 2 hours the sample was centrifuged at $4^{\circ} \mathrm{C}$, plasma separated and used for measurements or stored at $-20^{\circ} \mathrm{C}$ until analyzing.

IGF1 was measured using chemiluminiscence method (Liaison, Diasorin) (detection limit of 0.01 $\mathrm{ng} / \mathrm{mL}$, inter-assay CV $8.2 \%$ ) using monoclonal antibodies. Total P1NP was performed using monoclonal mouse antibodies against $\mathrm{P} 1 \mathrm{NP}$, using chemiluminescence method and a Cobas analyzer (Elecsys 2010 total P1NP, Roche Diagnostics- detection limit $5 \mathrm{ng} / \mathrm{mL}$, interassay $\mathrm{CV}<4.1 \%$ ). For $\mathrm{P} 1 \mathrm{NP}$, all the samples for any individual were measured in the same assay batch.

\section{Reference values}

We calculated age-specific means, standard deviations (SD) and the 95\% confidence intervals (CI) for IGF1 and P1NP using their values in the sGH group. For each of the two parameters, we assessed changes with age using a polynomial regression model (MedCalc v.14.8.1.0 statistical software). A second regression model gives the standard deviation as a function of age. We stratified $\mathrm{sGH}$ group into 2 age intervals (15-16.9 and 17-19 years), according to literature specifications for variation of P1NP values with age. To check for outliers at either side, we selected the Tukey-test.

We assessed the normality of the distribution using the Shapiro-Wilk's test for each age-stratified group. We assessed the homogeneity of variances using Levene's test. We calculated indices of skewness and kurtosis for each age group. We converted into standard deviations score (SDS) using the formula: $x$-average $x / S D$, where $x$ is the actual IGF 1 or P1NP of the patient, average $x$ is the mean IGF1 or P1NP and $S D$ is the standard deviation of the GH sufficient patients.

\section{Statistical methods}

Statistical analysis was performed using the SPSS v 16.0. Correlation between parameters was performed using Pearson's correlation. The statistical significance of the differences between the mean values among different categories was determined using the $t$ test (when comparing two 
groups). Two-sided tests of hypotheses were used, and a p-value $<0.05$ was considered statistically significant.

To evaluate the diagnostic performance of IGF1 and P1NP in the characterization of GHD in the transition period, we chose the cut-off of these parameters with the best specificity and sensitivity as resulted from ROC plot analysis. We used MedCalc v.14.8.1.0 statistical software to compare the performance of area under the curve (AUC) for the two parameters in identifying the GHD.

\section{RESULTS}

We evaluated seventeen male patients with GH (4 MPHD), mean age 17.3 years, treated during their childhood with recombinant $\mathrm{GH}$ for an average period of 6.88 years (range 2.9 to 12.4 years), after a minimum period of 3 months from the discontinuation of GH. Four of CO-IGHD patients after retesting during the transition maintained the GHD. An age-matched group of 8 controls together with the reversible GHD patients represent the GH sufficient group.

Table 1 shows clinical and biochemical characteristics of the subjects separated according to their GH status. All subjects had spontaneous puberty in Tanner stage $\mathrm{V}$ or adequate sex steroids substitution.

We stratified sGH group into 2 age intervals (15-16.9 and 17-19 years).

IGF1 and P1NP values had a normal distribution within the sGH group as shown by Shapiro-Wilk's test ( $p>0.05)$, the skewness of 0.835 [standard error (SE), 0.550], the kurtosis of $0.557(\mathrm{SE}=1.063)$ for IGF1, the skewness of $0.003(\mathrm{SE}=0.550)$ and the kurtosis of -0.459 ( $\mathrm{SE}=1.063$ ) for P1NP, respectively. The IGF1 and P1NP maintain the normal distribution in the age-stratified groups (data not shown). We used the sGH group as a reference for IGF1 and P1NP normal values.
We obtained for each year of age the mean and standard deviation (SD) for P1NP and IGF1 as a function of age. Age was a significant predictor for P1NP SD only.

We found a normal distribution for P1NP and IGF1 in the GHD group stratified by age.

We found an overlap between the absolute values of P1NP in GHD patients and the reference group. There was a trend towards a significant difference in concentrations of $\mathrm{P} 1 \mathrm{NP}$ when comparing the two groups $(p=0.07)$, with lower values in the $\mathrm{GH}$-deficient group.

The GH sufficient patients have higher P1NP SDS (mean $=0, \mathrm{SE}=0.23$ ) compared to GHD patients (mean $=-0.71, \mathrm{SE}=0.58$ ). This difference was significant $t(21)=2.24, \mathrm{p}<0.05$ and represent a medium sized effect $r=0.44$. None of P1NP SDS in the GHD group were below - 2SDS.

Although we did not find a significant difference between the means for the two parameters when we compared the group below 17 years age to the older group, we kept the age-stratified reference ranges due to the significant difference of SD.

Fig. 1 shows the relationship between the absolute level of P1NP and IGF1 using a scatter diagram. We found a strong positive correlation between the P1NP and IGF1 in GHD group as well as in $\mathrm{sGH}$ group $(\mathrm{r}=0.72,95 \% \mathrm{CI} 0.02$ to $0.94 ; \mathrm{P}=0.046$ and $\mathrm{r}=0.48,95 \% \mathrm{CI}-0.01$ to $0.79 ; \mathrm{P}=0.058$ respectively).

After we adjusted for chronological age, height deficit and weight, this correlation was maintained only by the GHD group. Table 2 shows correlations of P1NP and IGF1 with clinical parameters.

The ROC analysis showed that the best threshold for IGF1 SDS that discriminates between GHD and $\mathrm{sGH}$ was -1.34 SDS with a sensibility of $80 \%$ CI [44.4 to 97.5$]$, a specificity of $93.75 \%$ [69.8 to 99.8] and $\mathrm{AUC}=0.875$, CI [0.686 to 0.971]. The likelihood ratio for a positive test result $(\mathrm{LR}+)$ was

TABLE 1. Characteristics of the patients separated by $\mathrm{GH}$ status

\begin{tabular}{|l|c|c|c|c|}
\hline & \multicolumn{2}{|c|}{ pGHD $(\mathrm{n}=8)$} & \multicolumn{2}{c|}{$\mathrm{sGH}(17)$} \\
\hline BMI(SDS) & $0.40 \pm 1.82$ & {$[-2.9,1.4]$} & $-0.74 \pm 0.82$ & {$[-1.7,0.75]$} \\
\hline Ht (SDS) & $-1.1 \pm 1.12$ & {$[-3.2,0.09]$} & $-0.63 \pm 1.20$ & {$[-2.89,1.46]$} \\
\hline CA, years & $17.53 \pm 1.26$ & {$[15.2,18.9]$} & $16.94 \pm 1.01$ & {$[14.9,18.5]$} \\
\hline P1NP(SDS) & $-0.71 \pm 0.58$ & {$[-1.49,0.27]$} & $0 \pm 0.97$ & {$[-1.56,1.37]$} \\
\hline P1NP(ng/mL) & $214.36 \pm 74.57$ & {$[106.7,321.1]$} & $308.41 \pm 130.95$ & {$[120.2,537.8]$} \\
\hline IGF1(SDS) & $2.1 \pm 1.48$ & {$[-3.69,0.49]$} & $0 \pm 0.97$ & {$[-1.46,2.04]$} \\
\hline IGF1(ng/mL) & $209.68 \pm 151.88$ & {$[60.94,493.7]$} & $398.12 \pm 102.45$ & {$[250.8,638.8]$} \\
\hline timeTx (years) & $1.38 \pm 1.27$ & {$[0.3,4.2]$} & $0.81 \pm 0.53$ & {$[0.3,1.9]^{*}$} \\
\hline
\end{tabular}




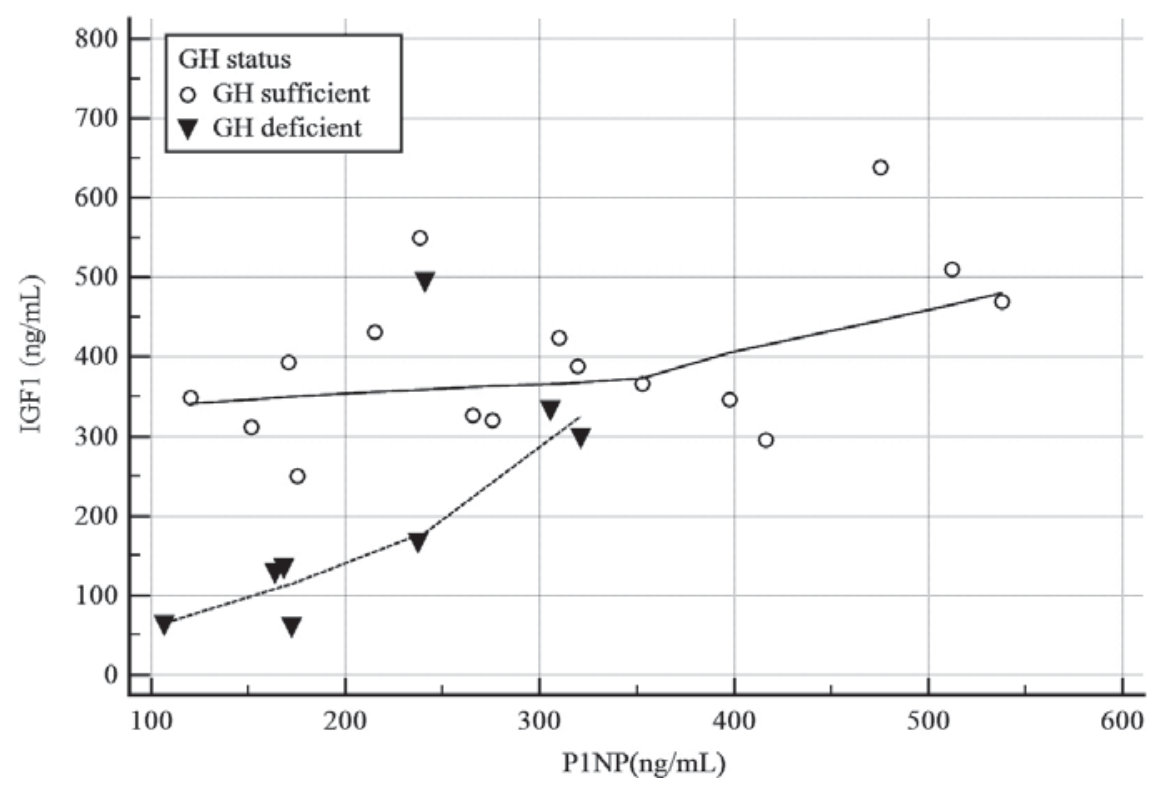

FIGURE 1. Correlation between amino-terminal propeptide type I procollagen (PINP) and insulin growth factor I(IGF1)

TABLE 2. Correlation of P1NP and IGF1 with clinical parameters

\begin{tabular}{|l|c|c|c|c|}
\hline & \multicolumn{2}{|c|}{ P1NP } & \multicolumn{2}{c|}{ IGF1 } \\
\hline & $r$ & $p$ & $r$ & $p$ \\
\hline Ht (SDS) & 0.424 & $\mathbf{0 . 0 3 9}$ & 0.338 & 0.098 \\
\hline Weight (kg) & -0.139 & 0.584 & -0.560 & $\mathbf{0 . 0 1 6}$ \\
\hline CA (years) & -0.383 & 0.065 & -0.593 & $\mathbf{0 . 0 0 2}$ \\
\hline timeTx (years) & -0.393 & 0.107 & -0.476 & $\mathbf{0 . 0 4 6}$ \\
\hline
\end{tabular}

$r$, Pearson's coefficient; P1NP, amino-terminal propeptide of type I procollagen; IGF1, insulin growth factor I; SDS, standard deviation score; Ht, height; CA, Chronological age; timeTX, time since growth hormone withdrawal; Significant $P$ values are listed in bold font.

12.8 [1.9 to 87.6]. The likelihood ratio for a negative test result (LR-) was 0.21 [0.06 to 0.7].

The ROC analysis showed the best threshold for P1NP SDS that discriminates between GHD and sGH was - 0.66 SDS with a sensibility of $62.5 \% \mathrm{CI}$ [24.5 to 91.5], a specificity of $75 \%$ [47.6 to 92.7 ] and $\mathrm{AUC}=0.719$; CI [0.5 to 0.881]. The likelihood ratio for a positive test result ( $\mathrm{LR}+$ ) was 2.5 [0.9 to 6.8]. The likelihood ratio for a negative test result (LR-) was 0.5 [0.2 to 1.3]. We did not find a significant difference when we compared the AUC for the two parameters $(\mathrm{p}=0.29)$.

\section{DISCUSSION}

P1NP is a marker of bone formation. It originates from the conversion of type I procollagen to type I collagen during his synthesis. The removal of the amino-terminal propeptide by specific proteases occurs when type I collagen is embedded in the bone matrix. P1NP concentration is related to the amount of newly formed bone (13). In 2011, the
International Osteoporosis Foundation and the International Federation of Clinical Chemistry and Laboratory Medicine Working group on Bone Marker Standards recommended P1NP use as a reference marker for bone formation (14).

Our data shows that, during the transition, P1NP is positively correlated with IGF1. Previous studies have shown that the lack of GH/IGF1 effect on bone turnover is associated with low collagen and bone formation during childhood, but not in adult life (12). The low serum procollagen propeptide in children with GHD has been linked with reduced growth velocity $(15,16)$. The subjects in our study were evaluated after attainment of final height as young adults, in a period when bone mineral acquisition continues.

We used a total P1NP assay that detects two different forms of antigenicity of P1NP, the trimeric, intact form and the monomeric form. This feature is particularly relevant when the changes to be measured are relatively small and would have expected a more dynamic behavior for an assay that 
detects the intact P1NP (13). The serum concentrations of P1NP in GHD patients were within the reference for normal individuals. This can be explained by two board reference intervals of these markers, with considerable inter-individual variation reflecting the high inter-individual variation of bone turnover in adults (17). Bone remodelling is usually balanced with approximately as much bone replaced as is removed during each remodelling cycle. However, when remodelling becomes accelerated in combination with an imbalance that favours bone resorption over formation, such as during menopause, precipitous losses in bone mass occur.

P1NP values vary by age. In our subjects, we found a negative correlation of P1NP with age because a decline of collagen marker levels is expected after puberty (18). Unlike the negative impact on IGF1 values, P1NP is unaffected by weight.

Similar values for P1NP between patients with reversible GHD and healthy controls suggest normal bone formation in the former, too.

\section{REFERENCES}

1. Molitch M.E., Clemmons D.R., Malozowski S., Merriam G.R. \& Vance, M.L. Evaluation and treatment of adult growth hormone deficiency: an Endocrine Society clinical practice guideline. J. Clin. Endocrinol. Metab. 96, 1587-609 (2011).

2. Mauras N., Walton P., Nicar M., Welch S. \& Rogol A.D. Growth hormone stimulation testing in both short and normal statured children: use of an immunofunctional assay. Pediatr. Res. 48 , 614-618 (2000).

3. Doessing S. et al. Growth hormone stimulates the collagen synthesis in human tendon and skeletal muscle without affecting myofibrillar protein synthesis. J. Physiol. 588, 341-51 (2010).

4. Erotokritou-Mulligan I. et al. The development of decision limits for the implementation of the $\mathrm{GH}-2000$ detection methodology using current commercial insulin-like growth factor-I and amino-terminal pro-peptide of type III collagen assays. Growth Horm. IGF Res. 22, 53-58 (2012).

5. Nguyen T.V. et al. Within-subject variability and analytic imprecision of insulinlike growth factor axis and collagen markers: implications for clinical diagnosis and doping tests. Clin. Chem. 54, 1268-76 (2008).

6. Ho K.K.Y. Consensus guidelines for the diagnosis and treatment of adults with GH deficiency II: a statement of the GH Research Society in association with the European Society for Pediatric Endocrinology, Lawson Wilkins Society, European Society of Endocrinology, J. Eur. J. Endocrinol. 157, 695-700 (2007).

7. Seibel M.J. The use of molecular markers of bone turnover in the management of patients with metastatic bone disease. Clin. Endocrinol. (Oxf). 68, 839-849 (2008).

8. Civitelli R., Armamento-Villareal R. \& Napoli N. Bone turnover markers: Understanding their value in clinical trials and clinical practice. Osteoporos. Int. 20, 843-851 (2009).

9. Schonau E. \& Rauch F. Markers of bone and collagen metabolismproblems and perspectives in paediatrics. Horm. Res. 48 Suppl 5 , 50-59 (1997).
We found a significant difference between P1NP expressed as SDS in GH-deficient patients when comparing with $\mathrm{GH}$ sufficient individuals.

This suggests a determinant role for GH-IGF1 axis during the transition, unlike in the adult life, when sex steroids have the primary effect on bone turnover markers (12). We calculated AUC of P1NP and we compared it to that of IGF1. We found a similar prediction power for $\mathrm{GH}$ deficiency for both parameters.

\section{CONCLUSION}

The collagen markers have less intra-individual variability in comparison to the IGF1. A marker with an increased coefficient of reliability is preferably used when quantifying the benefit during treatment. During the transition, when growth velocity is not anymore available, the dynamic of P1NP during GH replacement therapy, it may be useful in quantifying the effectiveness of GH treatment.

10. Crofton P.M. \& Kelnar C.J. Bone and collagen markers in paediatric practice. Int J Clin Pr. 52, 557-565 (1998).

11. Russell M., Breggia A., Mendes N., Klibanski A. \& Misra M. Growth hormone is positively associated with surrogate markers of bone turnover during puberty. Clin. Endocrinol. (Oxf). 482-488 (2011). doi:10.1111/j.1365-2265.2011.04088.x

12. Sartorio A., Conti A. \& Monzani M. New markers of bone and collagen turnover in children and adults with growth hormone deficiency. Postgrad. Med. J. 69, 846-850 (1993).

13. Koivula M.K., Risteli L. \& Risteli J. Measurement of aminoterminal propeptide of type I procollagen (PINP) in serum. Clin. Biochem. 45, 920-927 (2012).

14. Vasikaran S. et al. Markers of bone turnover for the prediction of fracture risk and monitoring of osteoporosis treatment: A need for international reference standards. Osteoporos. Int. 22, 391-420 (2011).

15. Carey D.E., Goldberg B., Ratzan S.K., Rubin K.R. \& Rowe D.W. Radioimmunoassay for type I procollagen in growth hormone-deficient children before and during treatment with growth hormone. Pediatr. Res. 19, 8-11 (1985).

16. Crofton P.M., Evans N., Taylor M.R.H. \& Holland C.V. Procollagen Type I Amino-Terminal Propeptide: Pedi- atric Reference Data and Relationship with Procollagen Type I Carboxyl-Terminal Propeptide. Clin. Chem. 50, 2173-2176 (2004).

17. Brown J.P. et al. Bone turnover markers in the management of postmenopausal osteoporosis. Clinical Biochemistry 42, 929-942 (2009).

18. Bayer M. Reference values of osteocalcin and procollagen type I $\mathrm{N}$-propeptide plasma levels in a healthy Central European population aged 0-18 years. Osteoporos. Int. 25, 729-736 (2014). 